\title{
Hat der Patient Sie auch verstanden?
}

\author{
Nicht nur ein Behandlungsfehler, auch eine mangelnde Auf- \\ klärung kann zu Schadenersatzklagen führen. Gerhard H. \\ Schlund, Experte für Arztrecht aus München, erläuterte in \\ Davos wichtige Aspekte einer angemessenen Aufklärung.
}

W ährend bei Behandlungsfehlern die Beweislast beim Patienten liegt, ist sie bei Aufklärungsfehlern Sache des Arztes. Deshalb ist die geeignete Dokumentation einer ausreichenden und verständlichen Aufklärung von größter Bedeutung. Juristisch ist schon eine Injektion eine Körperverletzung, die eine solche Aufklärung nötig macht. In der ambulanten Situation wird aber aus Zeitgründen meist darauf verzichtet. Schlund empfiehlt, die mündliche Aufklärung zumindest schriftlich in den Unterlagen zu vermerken („Aufklärung nach Standard“) und diesen Standard zu dokumentieren - auch gegenüber dem Praxisteam.

\section{Zeitabstand beachten}

Der Zeitfaktor spielt vor Gericht in Aufklärungsfragen immer eine große Rolle, berichtete Schlund. Dabei ist der zu fordernde Abstand zwischen Aufklärung und Beginn des Eingriffs von der Art des Eingriffs abhängig. Bei schweren Eingriffen, einem hohen Risiko und der Gefahr einer erheblichen Beeinträchtigung ist eine Aufklärung am Abend vorher zu spät. In diesem Fall sollten mindestens 24 Stunden zwischen Einwilligung und Operation liegen. Bei kleineren, z. B. ambulanten Eingriffen genügt eine kürzere Frist - aber Schlund empfiehlt auch hier, die Aufklärung mehrere Stunden vorher vorzunehmen und nicht direkt vor der Operation.

\section{Vom Umgang mit Aufklärungsbögen}

Der unterschriebene Aufklärungsbogen ist für die Dokumentation im Schadensfall wichtig und dreht die Beweislast um: Der Patient muss dann nachweisen, dass er nicht ausreichend aufgeklärt wurde. Vorgefertigte Aufklärungsbögen nennen bereits eine Vielzahl von Risiken und unterstützen damit die umfassende Auf- klärung. Treffen einige davon nicht zu, können diese gestrichen werden, um dem Patienten die Orientierung und das Verständnis des Bogens zu vereinfachen. Umgekehrt sollte die Aufklärung über besondere Risken, die bei einem speziellen Eingriff möglicherweise bestehen, in einem gesonderten Feld handschriftlich dokumentiert werden. Auch diese Angaben bestätigt der Patient dann mit seiner Unterschrift. Verzichtet ein Patient auf die umfassende Aufklärung, sollte das ausdrücklich in den Unterlagen vermerkt werden.

\section{Papier ersetzt nicht Gespräch}

Allerdings ist laut Schlund eine schriftliche Aufklärung mit Hilfe eines entspre-

\section{Uneinsichtige Autofahrer}

Typisch: Ein Patient kommt am Tag seiner ambulanten Operation mit dem eigenen Auto und ohne Begleitperson in die Praxis, obwohl er im Vorfeld darauf hingewiesen wurde, dass nach der Sedierung eine Teilnahme am Straßenverkehr nicht geboten ist. Im Gespräch sollte der Arzt dann vor dem Eingriff versuchen, den Patienten davon zu überzeugen, dass er den Autoschlüssel in der Praxis abgibt und mit dem Taxi oder öffentlichen Verkehrsmitteln nach Hause fährt.

In jedem Fall sollte aber der Patient vor dem Eingriff ein schriftliches Dokument unterzeichnen mit exakten Angaben, wie lange er nach dem Eingriff in der Praxis bleiben muss und dass er am Operationstag auf keinen Fall selbstständig Auto oder Motorrad fahren darf. Hält sich der Patient nicht an diese ernst gemeinten ärztlichen Ratschläge, dürfte das Haftungsrisiko des Arztes beschränkt sein. Denn einen geistig gesunden, aber aus ärztlicher Sicht unvernünftigen Menschen darf und kann man weder fixieren noch einschließen. $f k$ chenden Bogens nicht ausreichend, es muss auch ein Gespräch stattfinden. Dabei ist dafür zu sorgen, dass der Patient die erläuterten Risiken wirklich versteht. „Stellen Sie sich vor, der Patient hat BILD-Zeitungsniveau“, erläuterte Schlund. Deshalb sind Fremdwörter zu vermeiden und die Häufigkeit von Risiken nicht in Promille oder Prozent, sondern in Begriffskategorien wie „sehr selten“, „selten“, „gelegentlich“ usw. anzugeben.

Bei Patienten, die der deutschen Sprache nicht ausreichend mächtig sind, muss sich der Arzt entweder in der betreffenden Landessprache verständlich machen können oder eine Übersetzungshilfe (z. B. die Angehörigen) fordern. Bei elektiven Eingriffen kann der Arzt auch auf einem Dolmetscher bestehen, um eine angemessene Aufklärung sicher zu stellen. Die Kosten dafür muss der Patient selbst tragen. Bei Kindern bzw. senilen Patienten muss entsprechend ein Betreuer gefordert werden, der nach der Aufklärung in den Eingriff einwilligt.

\section{Unterlagen länger aufbewahren}

Rechtlich ist der Arzt verpflichtet, Patientenunterlagen wie z. B. Aufklärungsbögen 10 Jahre aufzubewahren, Röntgenunterlagen 30 Jahre. Schlund empfiehlt aber in allen Fällen eine Aufbewahrungszeit von 3 Jahrzehnten. Im Prozessfall, der oft erst viele Jahre nach der Behandlung eintritt, muss der Arzt auch nach mehr als 10 Jahren Unterlagen vorlegen können, die ihn entlasten und die Aufklärung und Behandlung dokumentieren.

$f k$

Schlund GH. Arzt und Aufklärungspflicht. 21. Fortbildungskongress „Fortschritte der Allergologie, Immunologie und Dermatologie“, Davos, 7.-10. September 2005 